\title{
Hemostasis Test Compatibility with Capped and Uncapped Tubes
}

\author{
KATHLEEN FINNEGAN, GWYN CUTSFORTH
}

OBJECTIVE: The objective of this study was to determine the substantial equivalence of routine and specialty hemostasis testing on capped versus uncapped evacuated blood collection tubes (3.2\% sodium citrate tubes, $2.7 \mathrm{ml}$ draw) on assays representing three most common assay types: viscosity, chromogenic, immunoturbidometric. Coagulation instrument manufacturers may provide a list of manufacturers and tube reference numbers that are compatible with their cappiercing technology on their instruments. However, the use of other blood collection tubes is the responsibility of the user and should be evaluated.

DESIGN: Seventy one volunteers donated two tubes (one each of two manufacturers) and the PT/INR, APTT, fibrinogen, AT and vWF assays were performed first on the capped tube, then on the same tube, uncapped. Assays were performed on the STACompact coagulation analyzer with reagents, kits and associated products (calcium chloride, DeSorb U, etc.) provided by Diagnostica Stago, Inc., Parsippany, New Jersey.

SETTING: The patient specimens were collected at the University Hospital at Stony Brook Outpatient Clinic. Assays were performed in the Stony Brook University, Department of Clinical Laboratory Science Teaching Laboratory.

PATIENTS/SPECIMENS: Informed consent was obtained from normal donors, individuals on oral anticoagulant therapy, ages 19 to 85 years, males and females. The Institutional Review Board approved this study (reference number 103249; Principle Investigator: K. Finnegan).

MAIN OUTCOME MEASURE: Singlicate values were compared between the samplings from capped and uncapped tubes by linear regression to determine the substantial equivalence. Singlicate measurements were made to maintain sample integrity to complete testing within four hours of the specimen draw.

RESULTS: Linear regression analysis and ANOVA demonstrated a strong correlation between capped and uncapped values with no bias. The correlation coefficient was greater than 0.763 for all assays and the regression line intercept was within acceptable error for the assays (see discussion, biological variation). The $\mathrm{p}$ value was greater than 0.08 for all assays.

CONCLUSIONS: The authors conclude that there is no significant difference between PT/INR, APTT and fibrinogen analysis on the capped or uncapped blood collection tubes tested in this study. Given the inherent biological variation of AT and $\mathrm{vWF}$, the authors conclude that there is no significant difference between AT and $\mathrm{WWF}$ analysis on the capped or uncapped blood collection tubes tested in this study.

ABBREVIATIONS: Prothrombin Time (PT), International Normalized Ratio (INR), Activated Partial Thromboplastin Time (APTT), fibrinogen (fib), antithrombin (AT) and von Willebrand Factor (vWF), State University of New York (SUNY), Becton Dickinson (BD), identification (ID), Greiner-Bio-One (GB1), milliliter $(\mathrm{ml})$, correlation coefficient $\left(\mathrm{R}^{2}\right)$

INDEX TERMS: cap-piercing, hemostasis testing, coagulation, capped tubes

Clin Lab Sci 2011;24(4):215

Kathleen Finnegan M.S. MT(ASCP)SH ${ }^{C M}$, SUNY Stony Brook University, Stony Brook, NY

Gwyn Cutsforth, MT(ASCP), PhD, Diagnostica Stago, Inc., Parsippany, NJ 


\section{RESEARCH AND REPORTS}

Address for Correspondence: Kathleen Finnegan M.S. $M T(A S C P) S H^{C M}$, Clinical Laboratory Sciences, School of Health Technology and Management, Level 2 Room 442 Health Sciences Center, 101 Nicolls Road, SUNY Stony Brook University, Stony Brook, NY 11794-8205, 631444-3224, kathleen.finnegan@stonybrook.edu

\section{INTRODUCTION}

Several manufacturers have introduced cap-piercing technology on their automated blood coagulation analyzers. This study was initiated to determine the compatibility of the Stago cap-piercing technology with tubes from two manufacturers that are not on the current tube compatibility list and to determine the substantial equivalence of routine hemostasis testing as well as two specialty coagulation tests on capped versus uncapped evacuated blood collection tubes. The prothrombin time (PT), International Normalized Ratio (INR), activated partial thromboplastin time (APTT) and fibrinogen (fib) are considered routine coagulation tests for this study; antithrombin (AT) and von Willebrand Factor (vWF) are considered specialty coagulation tests for this study. Manual methods of uncapping blood collection tubes expose laboratory staff to greater risk of exposure from aerosols and splashes and repetitive motion (ergonomic) injuries. ${ }^{1,2}$ The use of cap-piercing technology reduces laboratory staff exposure to blood borne pathogens and the repetitive motion of uncapping. However, cap-piercing technology must be evaluated to ensure that the technology functions correctly with the specific tube, a bias or additional variability is not introduced and to ensure the results are substantially equivalent between the capped and uncapped tube.

\section{MATERIALS and METHODS}

Volunteers were solicited from University Hospital Outpatient Clinic at the State University of New York (SUNY) Stony Brook presenting with physician orders for blood work. Seventy one volunteers donated two hemostasis collection tubes (one each of two manufacturers). Demographic data (age and sex) were collected and specimens were de-identified and assigned a unique study identification (ID). Demographic data (sex, age and anticoagulant therapy) were linked to the study ID. Specimens were drawn by routine venipuncture into $3.2 \%(0.109 \mathrm{M})$ tri-sodium citrate, $2.7 \mathrm{ml}$ vacuum blood collection tubes (Becton Dickinson, Franklin Lakes, New Jersey (BD) Item
\#363083 and Greiner-Bio-One, Monroe, North Carolina (GB1) Item \# 454334) (3,4 $^{3,4}$ by certified phlebotomists. Specimens were delivered to the Department of Clinical Laboratory Science Teaching Laboratory, processed for platelet poor plasma and tested by one individual within 4 hours of collection adhering to CLSI H21 A5 Collection, Transport, and Processing of Blood Specimens for Testing PlasmaCoagulation Assays and Molecular Hemostasis Assays; Approved Guideline, 2008. ${ }^{5}$

Assays were performed in singlicate first on the capped tube, then on the same tube, uncapped. The assays were performed on a Stago STA-Compact ${ }^{\oplus}$ with STA ${ }^{\oplus}$ System Controls N\&P, STA ${ }^{\oplus}$ Neoplastine CI+, STA ${ }^{\oplus}$ PTTA, STA ${ }^{\oplus}$-Fibrinogen, STA ${ }^{\oplus}$ Liatest $^{\oplus}$ Controls N\&P, STA Liatest ${ }^{\circ}$ VWF (Immuno-turbidimetric) and STA $^{\oplus}$ Stachrom ${ }^{\oplus}$ ATIII (Functional-Chromogenic) reagents. The PT, INR, APTT and fibrinogen test the viscosity-based detection system; AT and vWF tests the colorimetric and latex agglutination methods, respectively.

Linear regression analysis was performed by the SUNY Stony Brook School of Health Technology and Management, Department of Healthcare Policy and Management using Excel (Microsoft 2000-2003) and SPSS 17.0 (Windows 2009). ANOVA was also performed by David McGlasson at Wilford Hall Medical Center, Texas.

\section{RESULTS}

The values were compared between capped and uncapped by linear regression. Linear regression analysis demonstrated a strong correlation between capped and uncapped values $\left(\mathrm{R}^{2}>0.763\right)$ for all assays. Slope, intercept and $95 \%$ confidence intervals demonstrate that there is no significant difference between capped and uncapped tubes (BD or GB1) for the routine coagulation assays. Comparison of a sample's results of the specialty coagulation assays (AT and vWF) from capped versus uncapped tubes showed differences within the ranges defined for biological variation (www.westgard.com). ${ }^{6}$

The PT and INR values for the 71 samples tested were equivalent from both BD and GB1 tubes. The slopes and intercepts were approximately one indicating no bias or systematic difference between capped and uncapped tubes. APTT results also demonstrated a 


\section{RESEARCH AND REPORTS}

slope of essentially 1.0 and an intercept of 1 , implying a positive one second bias for the uncapped BD and GB1 tubes from the capped tubes. Fibrinogen results also demonstrated similar slopes $(1.0$ and 0.9$)$ and correlation coefficients $(0.9$ and 0.9$)$, however the intercepts were 24 and 43 for $\mathrm{BD}$ and Greiner tubes. The authors conclude that there is no difference in routine coagulation test results, using mechanical clot detection or viscosity, between capped and uncapped tubes, either BD or GB1.

The results for vWF and AT assay also do not demonstrate a difference between capped and uncapped tubes. The slopes are essentially one (0.9-1.1) and intercepts are similar. There were two samples from GB1 tubes whose vWF values were not similar between capped and uncapped (77 and 180; 127 and 67) however the differences were opposite in the direction of the difference. The samples were not repeated because the sample had aged past the 4 hour limit since blood draw.

Figure 1 presents the assay results obtained for both $\mathrm{BD}$ (Item \#363083) and GB1 (Item \# 454334) evacuated blood collection tubes sampled with cap-piercing technology (capped) and re-assayed from the same tube after manual removal of the cap (uncapped). The linear regression results are presented within the graphs and in Table 1.

The correlation coefficients range from 0.850 to 0.997 for the routine coagulation assays (PT/INR, APTT, fib) and from 0.760 to 0.981 for the specialty coagulations assays. The linear regression results, combined with the biological variation, demonstrate a good correlation between capped and uncapped assay results.

\section{DISCUSSION}

A search of the literature does not provide any published articles evaluating blood collection tubes with a cap-piercing technology. The implication is that without a published reference for a given manufacturer's tube and a given cap-piercing technology, the evaluation is being done independently in laboratories with independent protocols and acceptance criteria, potentially on the same products (tubes and technologies). Sharing of information through a published evaluation will serve as a basis for a laboratory to perform their own evaluation or to provide documentation for acceptance of a given blood collection tube and cap-piercing technology without the time and expense of performing the evaluation.

ANOVA analysis showed that the data from capped versus uncapped tubes were equivalent. ANOVA analysis is done when there is more than one variable and tests the hypothesis that the data sets are independent (i.e. similar or different) within and between data sets. Results ( $p$ value) range from zero to one and interpretation of the $95 \%$ confidence interval is a $p$ value less than 0.05 indicates that the data sets are different. ANOVA $\mathrm{p}$ values are presented with the regression analysis results in Table 1.

The results presented indicate that there is no difference in coagulation assay values between capped and uncapped tubes, thereby demonstrating acceptability of the tubes with the cap-piercing technology on the STA Compact $^{\oplus}$. The performance of an assay is dependent on the imprecision of the assay and is impacted by the biological variation of the measurand. Furthermore, the difference between two values is insignificant if it does not result in a change in the treatment of the patient. Evaluation of acceptability of test results is evaluated by the combination of the imprecision of the assay, the biological variation of the measurand and physician interpretation of the result.

\section{Biological Variation.}

In 2000, Dr. Carmen Ricos and colleagues published a database of biological variation for laboratory measurands. ${ }^{7}$ They have continued to update their data base, most recently in 2010 and the database is available on Dr. James Westgard's website. ${ }^{6}$ Biological variation is determined by the intra-individual and the interindividual variabilities. The accuracy and precision of a laboratory test should be taken in the context of the biological variation. A measurand with high biological variation would not require a high accuracy and precision assay to be able to interpret a change in the measurand. On the other hand, a measurand with low biological variation would require a high accuracy and precision assay to interpret a change in the measurand.

Total allowable error for the prothrombin time is 5.3\%, meaning that the prothrombin time accuracy and precision should be no more than approximately \pm 0.5 seconds. 7 Therefore, in comparing the capped tube prothrombin time versus the uncapped prothrombin 


\section{RESEARCH AND REPORTS}
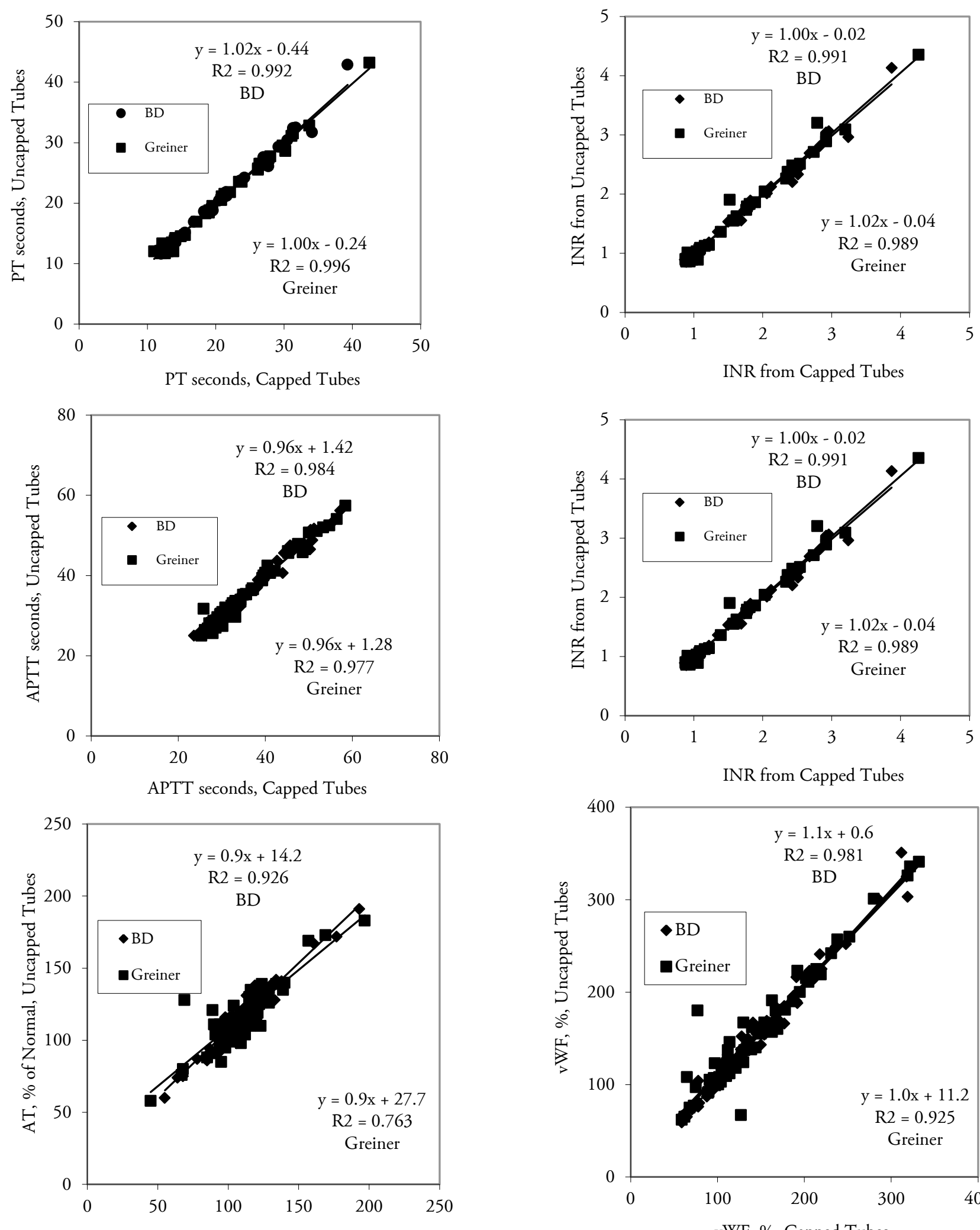

AT, \% of Normal, Capped Tubes

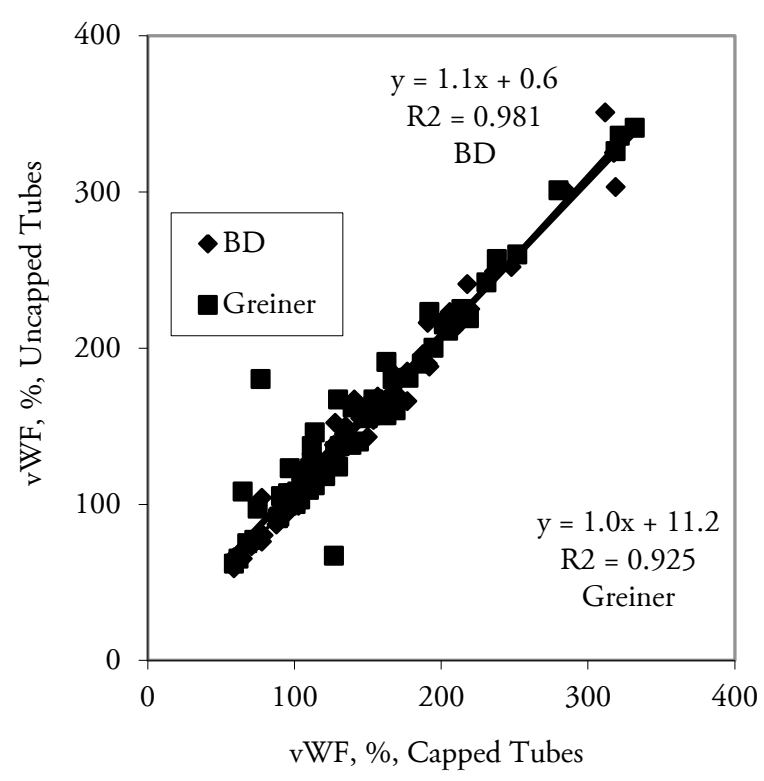

Figure 1. Assay results for BD and GB1. 
RESEARCH AND REPORTS

Table 1. Linear Regression and ANOVA Analysis Results

\begin{tabular}{|c|c|c|c|c|c|c|c|c|c|}
\hline \multicolumn{4}{|c|}{ 2.7ml BD tubes (Item \#363083) } & \multicolumn{3}{|c|}{ 2.7 ml GB1 tubes (Item \#454334) } & \multicolumn{3}{|c|}{ ANOVA analysis } \\
\hline Assay & Slope & $\begin{array}{c}\mathrm{N}=71 \\
\text { Intercept }\end{array}$ & $\mathrm{R} 2$ & Assay & Slope & $\begin{array}{c}\mathrm{N}=71 \\
\text { Intercept }\end{array}$ & $\mathrm{R} 2$ & Assay & $\begin{array}{l}\mathrm{N}=284 \\
\text { P value* }^{*}\end{array}$ \\
\hline PT & 1.02 & -0.44 & 0.992 & PT & 1.00 & -0.24 & 0.996 & PT & 0.996 \\
\hline INR & 1.00 & -0.02 & 0.991 & INR & 1.02 & -0.04 & 0.989 & INR & 0.998 \\
\hline APTT & 0.96 & 1.42 & 0.984 & APTT & 0.96 & 1.28 & 0.977 & APTT & 0.996 \\
\hline FIB & 1.0 & 24.3 & 0.951 & FIB & 0.9 & 43.0 & 0.882 & FIB & 0.380 \\
\hline AT & 0.9 & 14.2 & 0.926 & AT & 0.9 & 27.7 & 0.763 & AT & 0.080 \\
\hline vWF & 1.1 & 0.6 & 0.981 & vWF & 1.0 & 11.2 & 0.925 & vWF & 0.776 \\
\hline
\end{tabular}

${ }^{*} \mathrm{p}<0.05$ is statistically significant for a difference in the compared data sets.

time, the values would not be considered different if the values agreed within \pm 1 second. Greater than $95 \%$ of the samples tested (see Figure 1) did not show values differing by more than one second between analysis of the capped versus uncapped blood collection tube.

The APTT gives similar results to the PT with a total allowable error of $4.5 \% .^{7}$ Fibrinogen assays have a recommended total allowable error of $13.6 \% .^{7}$ This relatively large variation also incorporates the role of fibrinogen as an acute phase reactant. Antithrombin and $\mathrm{vWF}$ assays have similar total allowable errors, $8.3 \%$ and $7.1 \%$, respectively. ${ }^{7}$ The wide normal range of AT expressed as per cent of normal contributes to the high error as well as the multiple sources of $\mathrm{vWF}$ (platelets, endothelial cells, etc) contributing to the total concentration increases the allowable error of the measurements.

Given the inherent biological variations of the routine and specialty coagulation assays, our conclusion is that there is no difference between sample analyses on the capped or uncapped blood collection tubes tested in this study.

\section{Assay Value Ranges}

The results obtained from the volunteer blood donors were over a broader range than normal values. The samples from anti-vitamin $\mathrm{K}$ therapy patients provided PT and INR values up to approximately three-fold greater than the normal range for PT and INR. The range of values for APTT was approximately double the range expected for normals. Fibrinogen, AT and vWF showed the most variation in assay value ranges between capped and uncapped tubes, for the same reasons as discussed with biological variation. The assayed ranges for the specimens collected in this study are presented in Table 2. Table 2 also presents published normal ranges for the assays as a reference. ${ }^{8}$

\section{Demographic Analysis}

Statistical analysis of demographic subgroups was not done due to the small sample size. However, it can be said that these subgroups could not be differentiated from the full data set. The age range of the donors was from 19 to 85 years of age. Blood specimens were contributed from 29 males and 42 females. The study assayed a total of 23 samples from patients on antivitamin $\mathrm{K}$ therapy (17 men, 6 female).

\section{CONCLUSION}

In summary, both BD and GB1 tubes gave similar assay results, whether the coagulation assays were performed using the cap-piercing technology (capped tube) or the manually-opened (uncapped) evacuated blood collection tubes. Laboratories should verify the equivalence of sampling from capped and uncapped tubes to determine the compatibility of the blood collection tubes used at their institution with the cappiercing technology on their instrument. The results should be evaluated with the knowledge of the assay imprecision, the biological variation of the measurand and the physician interpretation of a change in the result.

This research project was approved by SUNY Stony Brook Institutional Review Board, effective date June 23, 2009.

\section{ACKNOWLEDGEMENT}

The authors would like to express their appreciation to 
Table 2. Assay value ranges

\begin{tabular}{lccccc}
\hline \multicolumn{2}{c}{ BD } & \multicolumn{2}{c}{ Greiner } \\
\hline \multicolumn{1}{c}{ Assay } & $\begin{array}{c}\text { Capped } \\
\text { Range }\end{array}$ & $\begin{array}{c}\text { Uncapped } \\
\text { Range }\end{array}$ & $\begin{array}{c}\text { Capped } \\
\text { Range }\end{array}$ & $\begin{array}{c}\text { Uncapped } \\
\text { Range }\end{array}$ & $\begin{array}{c}\text { Normal } \\
\text { Ranges }\end{array}$ \\
\hline PT, seconds* & $11.8-39.3$ & $11.6-42.9$ & $11.0-42.5$ & $11.7-43.2$ & $11-13$ \\
INR* & $0.9-3.87$ & $0.9-4.13$ & $0.9-4.26$ & $0.9-4.35$ & $0.8-1.2$ \\
APTT, seconds & $23.6-57.2$ & $25.0-56.2$ & $25.3-58.4$ & $25.0-57.2$ & $200-400$ \\
Fibrinogen, mg/dl & $147-665$ & $156-700$ & $145-729$ & $53-700$ & $80-125$ \\
AT, \% & $55-193$ & $60-191$ & $45-197$ & $62-341$ & $42-139$ \\
vWF, \% & $59-319$ & $62-341$ & $59-420$ & & \\
\hline
\end{tabular}

* includes patients on anti-vitamin $\mathrm{K}$ therapy

David McGlasson for helpful discussions and for ANOVA analysis of the data.

\section{REFERENCES}

1. OSHA Regulation 29CFR 1910.1030 Blood Borne Pathogens

2. Iskarous, R. Decap the Safe, Ergonomic and Efficient Way. Advance for Administrators of the Laboratory 2005;14(7):109.

3. Becton Dickinson Product Catalog: Available from www.catalog.bd.com/bdCat/listsmallProduct.doCUSTOMER? productnumber $=363083$. Accessed 2010December 16

4. Greiner - bio- one Vacuette Coagulation Tubes: Available from www.gbo.com/documents/980136_Gerinnungsbroschuer e_e_rev00_1009_small.pdf. Accessed 2010December16
5. CLSI H21 A5 Collection, Transport, and Processing of Blood Specimens for Testing Plasma-Coagulation Assays and Molecular Hemostasis Assays; Approved Guideline, Fifth Edition, 2008.

6. Westgard QC: Available from www.Westgard.com. Accessed 2010December 16

7. Ricós C, Alvarez V, Cava F, García-Lario JV, Hernández A, Jiménez CV, et al. Current databases on biological variation: pros, cons and progress. Scan J Clin Lab Invest 1999;59:475586

8. McKenzie SB, Williams JC, editors. Clinical Laboratory Hematology, $2^{\text {nd }}$ Edition, Saddle River, New Jersey, Pearson, 2010 .

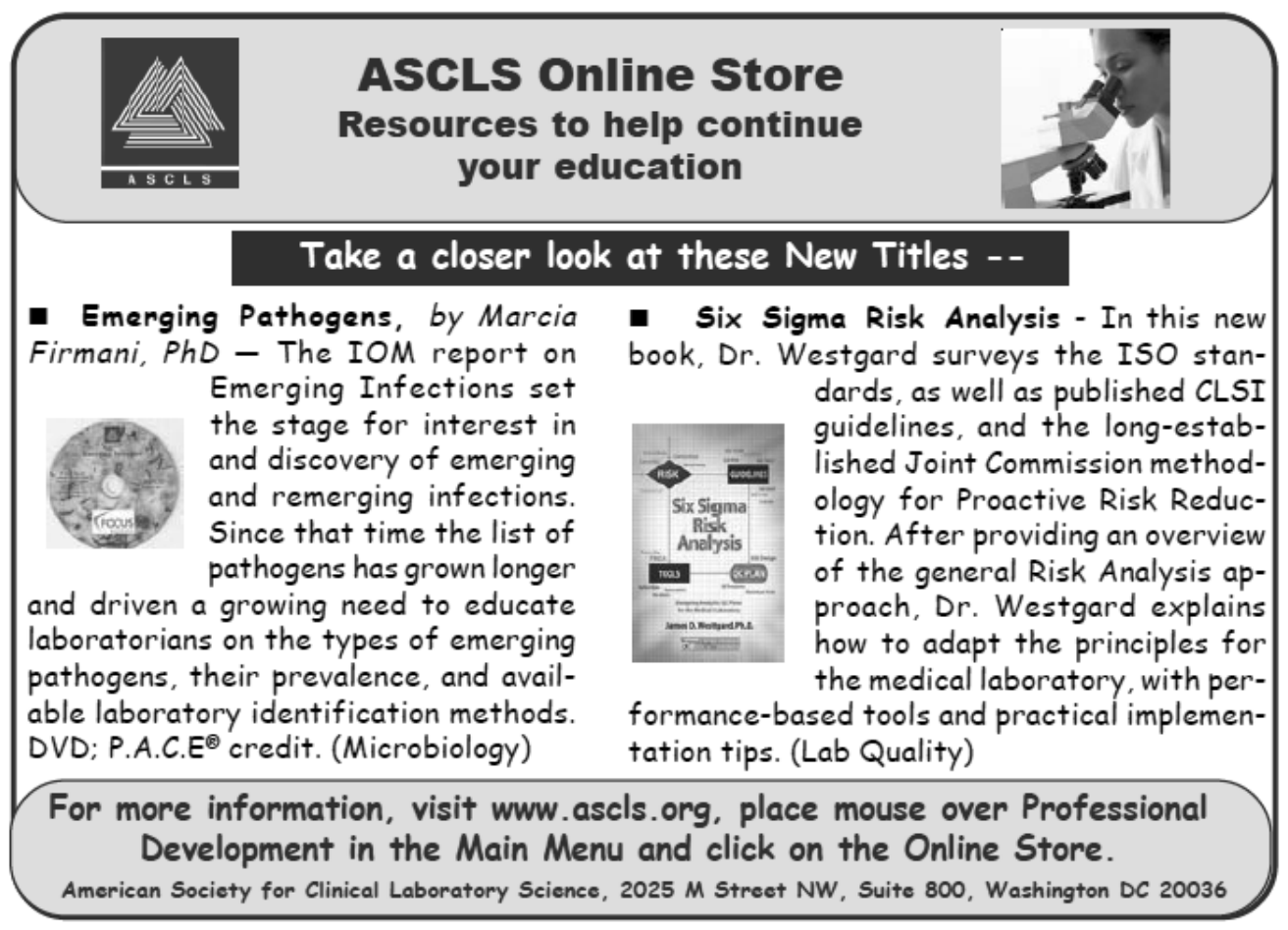

